\title{
The inplane elastic properties of circular cell and elliptical cell honeycombs
}

\author{
J. Clung and A. M. Waas, Ann Arbor, MI \\ (Received April 2, 1999; revised August 16, 1999)
}

Summary. The inplane elastic properties of perfectly circular and elliptic cell honeycombs are derived through an analytical method and validated numerically. In the case of perfectly circular cell hexagonally packed honeycomb, the inplane elastic properties are shown to be isotropic. However, a departure from circularity of the cells leading to cell ellipticity results in the inplane properties becoming orthotropic. The orthotropic elastic constants are also derived analytically and validated numerically.

\section{Introduction}

Solids with cellular microstructure are finding increased applications in the design of light weight structures. Cellular solids range from hexagonal celled bee's honeycombs to disordered three-dimensional skeletal networks of foams and sponges. Gibson and Ashby [1] is an excellent introduction to the subject of contemporary cellular solids. Because of their usage as structural load bearing components, there is a strong need to understand the mechanisms of deformation and failure of cellular solids. In particular, there is a need to obtain macroscopic ${ }^{1}$ structural properties of a cellular solid in terms of the properties of the smallest repeating unit of the solid, which we term a perfect unit representative cell (PURC). In reality, such PURC may not exist, since all of the cells (or clusters of cells) in a cellular solid may not be identical.

Previous work aimed at studying the mechanical properties of honeycombs and foam is amply summarized in the text of Gibson and Ashby [1]. These researchers and other groups elsewhere, Warren and Kraynik [2], Kyriakides and Papka [3], [4], Klintworth and Stronge [5], Triantafyllidis and Schraad [6], Gibson et al. [7], [8] have all investigated several aspects of the deformation and failure of particular classes of cellular solids.

In the present paper, we are concerned with obtaining closed form solutions to the elastic properties of a class of circular cell and elliptical cell space filling honeycombs. Figure 1 shows a circular cell honeycomb with a set of rectangular cartesian axes $X-Y-Z$ depicted to identify the different planes of the honeycomb. When all the cells in the $X-Y$ plane are identical, then such a hexagonally packed circular cell honeycomb displays isotropic behavior in the $X-Y$ plane. If the cells are identical but elliptic, then the $X-Y$ plane is orthotropic. In the former case (circular cells), the entire solid is termed transversely isotropic and one needs five independent elastic constants to fully characterize its $3 D$ macroscopic stress-strain relation. In the latter case (elliptic cells), the macroscopic solid is orthotropic and one needs nine independent elastic constants to fully characterize its $3 D$ stress-strain response.

\footnotetext{
1 By macroscopic, we mean a volume of solid that occupy several tens of cells.
} 


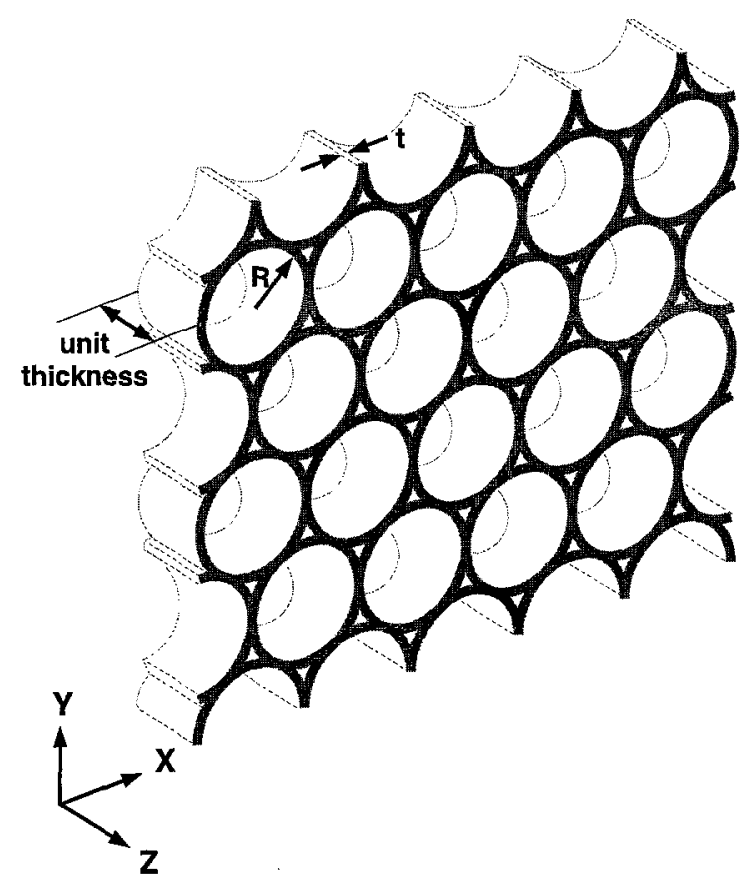

Fig. 1. A hexagonally packed circular cell honeycomb in three-dimensional view

The paper is organized as follows. We first treat the perfectly circular cell case and derive expressions for the inplane (the $X-Y$ plane) Young's moduli, the major Poisson's ratio ${ }^{2}$ and the shear modulus. We next show that the derived shear modulus satisfies the well known formula for an isotropic solid

$G_{x y}=\frac{E}{2\left(1+\nu_{x y}\right)}$.

Next, we observe that a rotation of the $X-Y$ coordinates by $\pi / 3$ results in an identical microstructure of the honeycomb and hence we conclude that the $X-Y$ plane is a plane of isotropy. Next, we derive the four elastic constants of a honeycomb with perfectly elliptical cells. Finally, we identify a small parameter $(\varepsilon)$ associated with slight ellipticity of the cells and obtain closed form expressions for elastic properties of such a honeycomb in terms of this small parameter. We also show that the results for elliptical cell honeycombs reduce to the closed form expressions obtained for the circular cell honeycombs in the limit of vanishing $\varepsilon$.

\section{Inplane properties of perfect circular cell hexagonally packed honeycombs}

Figure 2 shows a PURC of the circular cell honeycomb. Consider the response of the honeycombs to three different inplane loading programs taken separately, one at a time. Then, by modeling the cell walls within the context of classical thin walled Euler-Bernoulli beam theory and using Castigliano's theorem [9], it is possible to arrive at an expression for the different elastic moduli corresponding to the particular loading program. Kyriakides and Papka [4]

\footnotetext{
2 In an orthotropic solid, one can define, per plane, 2 Poisson's ratios, 2 Young's moduli and a shear modulus. Of these, only four quantities are independent since the poisson's ratios satisfy the reciprocity relation $\left(E_{x} \nu_{y x}=E_{y} \nu_{x y}\right)$.
} 


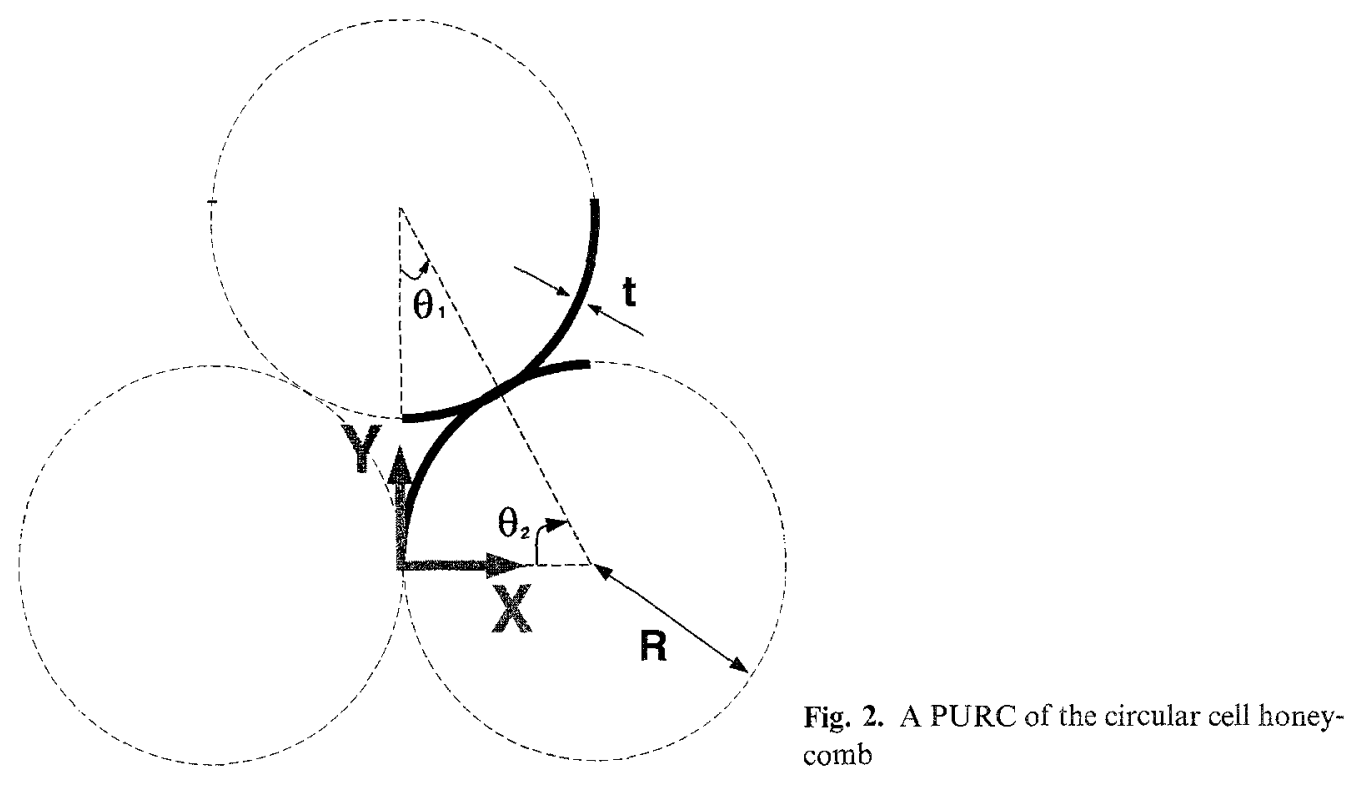

used this approach to obtain the Young's modulus of a circular cell hexagonally packed honeycomb with the initial assumption of macroscopic isotropy.

The two Young's moduli $E_{x}{ }^{*}$ and $E_{y}{ }^{*}$, the shear modulus $G_{x y}^{*}$ and the inplane Poisson's ratio $\nu_{x y}^{*}$ are calculated as illustrated in Fig. 3.

In Fig. $3 \mathrm{a}$, we show the free body diagram of a PURC when subjected to a macroscopic stress $\sigma_{x}$ in the $X$-direction. Symmetry with respect to loading and geometry was used in arriving at the free body diagram indicated. Note that $P_{1}$ and $P_{2}$ are externally applied forces and not the internal resultants. Within the context of classical beam bending theory (neglecting any membrane deformation of the cell walls), the elastic strain energy of the PURC is

$U=2\left[\int_{0}^{\pi / 6} \frac{\left[M_{1}\left(\theta_{1}\right)\right]^{2}}{2 \hat{D}} R d \theta_{1}+\int_{0}^{\pi / 3} \frac{\left[M_{2}\left(\theta_{2}\right)\right]^{2}}{2 \hat{D}} R d \theta_{2}\right]$,

where, $M_{1}\left(\theta_{1}\right)$ and $M_{2}\left(\theta_{2}\right)$ are the moment intensity distributions in sector $k_{1} k_{2}$ and $k_{3} k_{2}$, respectively. $\theta_{1}$ and $\theta_{2}$ are coordinates as indicated in the figure.

$\hat{D}=\frac{E^{\prime} t^{3}}{12}$

where

$E^{\prime}=\frac{E}{1-\nu^{2}}$.

In the above equation, $E$ and $\nu$ indicate the Young's modulus and the Poisson's ratio of the honeycomb material, respectively.

In order to calculate the honeycomb macroscopic elastic modulus $E_{x}{ }^{*}$ in the $X$-direction, we invoke Castigliano's theorem to arrive at

$\frac{\partial U}{\partial M_{a}}=0, \quad \frac{\partial U}{\partial P_{1}}=\delta, \quad \frac{\partial U}{\partial P_{2}}=\delta$. 


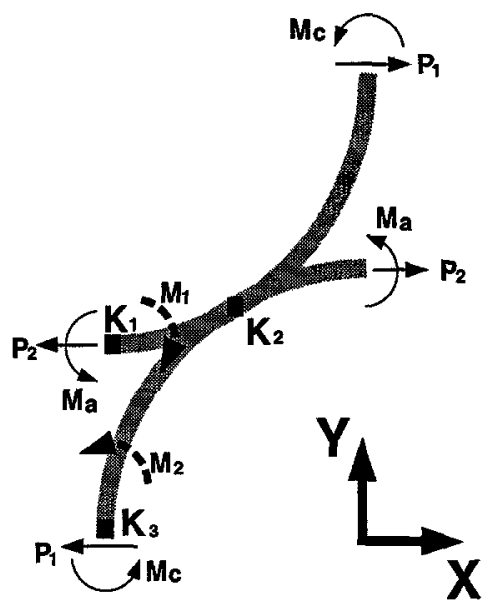

a

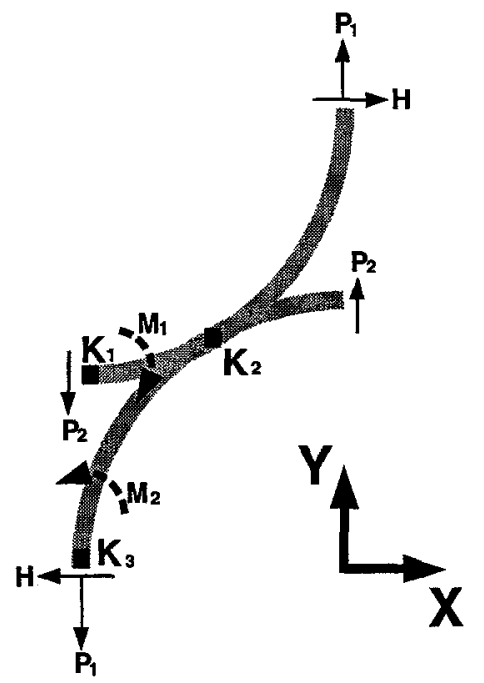

c

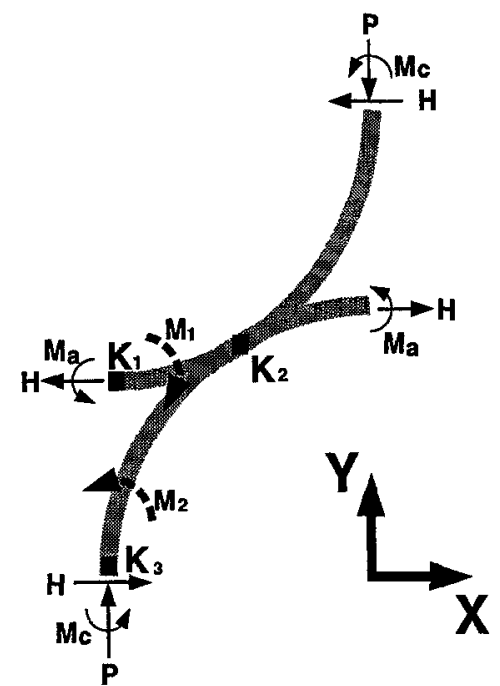

b

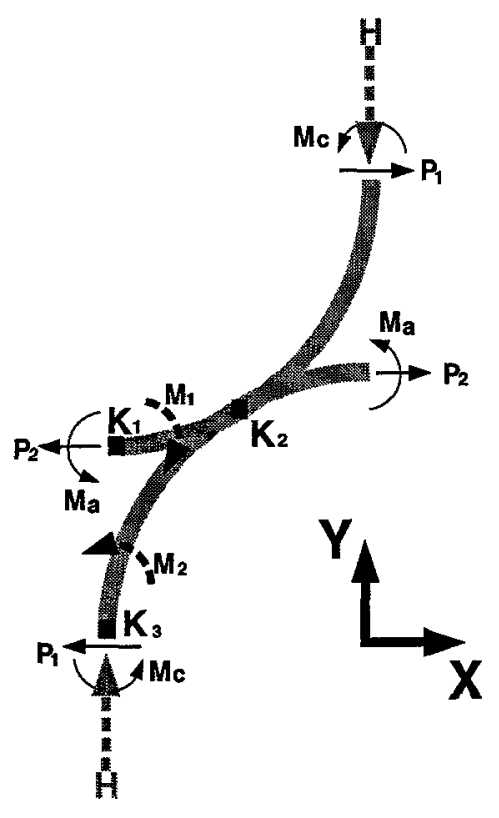

d

Fig. 3. a Free body diagram of circular PURC used to calculate the elastic modulus in the $X$-direction; b Free body diagram of circular PURC used to calculate the elastic modulus in the $Y$-direction; c Free body diagram of circular PURC used to calculate the shear modulus in the $X-Y$ plane; $\mathbf{d}$ Free body diagram of circular PURC used to calculate the Poisson's ratio in the $X-Y$ plane

In evaluating $U$, overall equilibrium is first satisfied so that $M_{c}$ can be expressed in terms of $M_{a}, P_{1}$ and $P_{2}$. Next, general expressions for $M_{1}\left(\theta_{1}\right)$ and $M_{2}\left(\theta_{2}\right)$ in terms of $M_{a}, P_{1}$ and $P_{2}$ are obtained and substituted in the expression for $U$. Using (2), we are able to compute $P_{1}$ and $P_{2}$ as a linear function of $\delta$.

The Young's modulus parallel to the $X$ axis is $E_{x}{ }^{*}=\left(\sigma_{x} / \varepsilon_{x}\right)$, giving $\frac{E_{x}^{*}}{E} \approx \frac{8.329}{\left(1-\nu^{2}\right)}\left(\frac{t}{R}\right)^{3}$ 
Note that an $*$ is used to indicate macroscopic honeycomb properties. Using a relative density (the density, $\varrho^{*}$, of the honeycomb structure divided by the density, $\varrho$, of the material the honeycomb structure is made of), the above equation becomes

$\frac{E_{x}^{*}}{E} \approx \frac{1.396}{\left(1-\nu^{2}\right)}\left(\frac{\varrho^{*}}{\varrho}\right)^{3}$

Loading in the $Y$-direction and the corresponding PURC is shown in Fig. 3 b. In Fig. 3 b, $P$ is the externally applied force and not the internal resultant. This case is similar to that reported in Papka and Kyriakides [4]. The strain energy is

$U=2\left[\int_{0}^{\pi / 6} \frac{\left[M_{1}\left(\theta_{1}\right)\right]^{2}}{2 \hat{D}} R d \theta_{1}+\int_{0}^{\pi / 3} \frac{\left[M_{2}\left(\theta_{2}\right)\right]^{2}}{2 \hat{D}} R d \theta_{2}\right]$.

Invoking Castigliano's theorem, the following equations are obtained.

$\frac{\partial U}{\partial M_{a}}=0, \quad \frac{\partial U}{\partial H}=0, \quad \frac{\partial U}{\partial P}=\delta$.

The above equations in conjunction with overall force and moment equilibrium of the corresponding PURC results in

$\frac{E_{y}^{*}}{E} \approx \frac{8.329}{\left(1-\nu^{2}\right)}\left(\frac{t}{R}\right)^{3}$

In terms of the relative density, the above equation becomes

$\frac{E_{y}{ }^{*}}{E} \approx \frac{1.396}{\left(1-\nu^{2}\right)}\left(\frac{\varrho^{*}}{\varrho}\right)^{3}$.

To calculate the inplane shear modulus $G_{x y}^{*}$, we use a pure shear loading, whence the corresponding free body diagram of the PURC is as shown in Fig. $3 \mathrm{c}$. In Fig. $3 \mathrm{c}$, note that $P_{1}, P_{2}$ and $H$ are externally applied forces and not the internal resultants. The expression for strain energy $U$ is given by

$U=2\left[\int_{0}^{\pi / 6} \frac{\left[M_{1}\left(\theta_{1}\right)\right]^{2}}{2 \hat{D}} R d \theta_{1}+\int_{0}^{\pi / 3} \frac{\left[M_{2}\left(\theta_{2}\right)\right]^{2}}{2 \hat{D}} R d \theta_{2}\right]$.

With respect to Fig. $3 \mathrm{c}$, noting that $M_{1}\left(\theta_{1}\right)=P_{2} R \sin \theta_{1}, M_{2}\left(\theta_{2}\right)=H R \sin \theta_{2}-P_{1} R$ $\times\left(1-\cos \theta_{2}\right)$ and invoking overall moment equilibrium, with the latter resulting in $H=\left(P_{1}+P_{2}\right) / \sqrt{3}, U$ as given in (5) is evaluated. Then, Castigliano's theorem delivers the following two conditions:

$\frac{\partial U}{\partial P_{1}}=\Delta \quad$ and $\quad \frac{\partial U}{\partial P_{2}}=\Delta$

The above condition (6) in conjunction with the definition (5), provides the following result for $G_{x y}^{*}$, which is defined as

$G_{x y}^{*}=\frac{\tau}{\gamma}=\frac{P_{1}+P_{2}}{2 \Delta \sin \frac{\pi}{3}}$. 
Thus,

$\frac{G_{x y}^{*}}{E} \approx \frac{2.306}{\left(1-\nu^{2}\right)}\left(\frac{t}{R}\right)^{3}$

In terms of the relative density, the above equation reduces to

$\frac{G_{x y}^{*}}{E} \approx \frac{0.386}{\left(1-\nu^{2}\right)}\left(\frac{\varrho^{*}}{\varrho}\right)^{3}$

In order to calculate the macroscopic Poisson's ratio $\nu_{x y}^{*}$, we use a circular PURC subjected to a macroscopic stress $\sigma_{x}$ in the $X$-direction and a virtual macroscopic stress $\sigma_{y}$ in the $Y$-direction caused by a virtual force $H$. The corresponding free body diagram is shown in Fig. $3 \mathrm{~d}$. Note that $P_{1}$ and $P_{2}$ are externally applied real forces and $H$ is the externally applied virtual force and they are not the internal resultants. The expressions for the internal moments $M_{1}\left(\theta_{1}\right)$ and $M_{2}\left(\theta_{2}\right)$ are

$M_{1}\left(\theta_{1}\right)=-M_{a}-P_{2} R\left(1-\cos \theta_{1}\right)$,

and

$M_{2}\left(\theta_{2}\right)=M_{c}+P_{1} R \sin \theta_{2}+H R\left(1-\cos \theta_{2}\right)$.

Overall equilibrium supplies

$M_{c}=-M_{a}+P_{2} R\left(\sin \frac{\pi}{3}-1\right)-P_{1} R \sin \frac{\pi}{3}-\frac{1}{2} H R$.

With $U$ defined as

$U=2\left[\int_{0}^{\pi / 6} \frac{\left[M_{1}\left(\theta_{1}\right)\right]^{2}}{2 \hat{D}} R d \theta_{1}+\int_{0}^{\pi / 3} \frac{\left[M_{2}\left(\theta_{2}\right)\right]^{2}}{2 \hat{D}} R d \theta_{2}\right]$

and invoking Castigliano's theorem, we obtain

$$
\begin{aligned}
\frac{\partial U}{\partial M_{a}}=0 & \frac{\partial U}{\partial H}=-\Delta, \\
\frac{\partial U}{\partial P_{1}}=\delta & \frac{\partial U}{\partial P_{2}}=\delta .
\end{aligned}
$$

After a lengthy manipulation of (7), taking the limit $H \rightarrow 0$, and with the definition of $\nu_{x y_{3}}^{*}=-\varepsilon_{y} / \varepsilon_{x}=-\left[1 /\left(2 \sin \frac{\pi}{3}\right)\right](\Delta / \delta)$, we obtain

$\nu_{x y}^{*} \approx 0.806$.

With respect to the Cartesian $X-Y$ system, the macroscopic stress-strain relation of the honeycomb is

$$
\left[\begin{array}{c}
\sigma_{x} \\
\sigma_{y} \\
\sigma_{x y}
\end{array}\right]=\left[\begin{array}{ccc}
\frac{E_{x}{ }^{*}}{1-\nu_{x y}^{*} \nu_{y x}^{*}} & \frac{\nu_{x y}^{*} E_{y}{ }^{*}}{1-\nu_{x y}^{*} \nu_{y x}^{*}} & 0 \\
\frac{\nu_{y x}^{*} E_{x}{ }^{*}}{1-\nu_{x y}^{*} \nu_{y x}^{*}} & \frac{E_{y}{ }^{*}}{1-\nu_{x y}^{*} \nu_{y x}^{*}} & 0 \\
0 & 0 & G_{x y}^{*}
\end{array}\right]\left[\begin{array}{c}
\varepsilon_{x} \\
\varepsilon_{y} \\
\gamma_{x y}
\end{array}\right]
$$


Table 1. Comparison of material constants between analytical solution and numerical simulation of PURC

\begin{tabular}{|c|c|c|c|}
\hline $\begin{array}{l}\text { Aspect ratio } \\
\text { of PURC } \\
a / b\end{array}$ & $\begin{array}{l}\text { Analytical solution } \\
\text { of PURC } \\
E_{x}^{*}, E_{y}{ }^{*}, G_{x y}^{*}(\mathrm{KPa}) \\
\nu_{x y}^{*}\end{array}$ & $\begin{array}{l}\text { Numerical Simulations } \\
\text { of PURC } \\
E_{x}^{*}, E_{y}{ }^{*}, G_{x y}^{*}(\mathrm{KPa}) \\
\nu_{x y}^{*}\end{array}$ & $\begin{array}{l}\text { Difference between } \\
\text { Column (1) and Column (2) } \\
\Delta E_{x}{ }^{*} \Delta E_{y}{ }^{*}, \Delta G_{x y}^{*}(\mathrm{KPa}) \\
\Delta \nu_{x y}^{*}\end{array}$ \\
\hline $\begin{array}{l}1 \\
\text { (circie) }\end{array}$ & $\begin{array}{l}751,751,208 \\
0.806\end{array}$ & $\begin{array}{l}752,752,208 \\
0.807\end{array}$ & $\begin{array}{l}1,1,0 \\
0.001\end{array}$ \\
\hline $\begin{array}{l}0.9596 \\
\text { (min. ellipticity) }\end{array}$ & $\begin{array}{l}701,817,206 \\
0.745\end{array}$ & $\begin{array}{l}702,817,206 \\
0.746\end{array}$ & $\begin{array}{l}1,0,0 \\
0.001\end{array}$ \\
\hline $\begin{array}{l}1.042 .7 \\
\text { (average ellipticity) }\end{array}$ & $\begin{array}{l}805,690,210 \\
0.873\end{array}$ & $\begin{array}{l}805,690,210 \\
0.873\end{array}$ & $\begin{array}{l}0,0,0 \\
0\end{array}$ \\
\hline $\begin{array}{l}1.1129 \\
\text { (max. ellipticity) }\end{array}$ & $\begin{array}{l}894,602,212 \\
0.987\end{array}$ & $\begin{array}{l}896,604,213 \\
0.987\end{array}$ & $\begin{array}{l}2,2,1 \\
0\end{array}$ \\
\hline
\end{tabular}

If we transfer (8) to a system of axes $\hat{X}-\hat{Y}$ that is inclined at $\Pi / 3$ to the $X-Y$ system, then the honeycomb stress-strain relation corresponding to (8), in this new coordinate frame is identical to (8) provided that

$E_{x}^{*}=E_{y}^{*} \quad$ and $\quad G_{x y}^{*}=\frac{E_{x}^{*}}{2\left(1+\nu_{x y}^{*}\right)}$.

These equalities are clearly satisfied by the expressions we derived earlier. Thus, the perfectly circular cell honeycomb is macroscopically transversely isotropic, with the $X-Y$ plane being the plane of isotropy.

The analytically derived results were checked numerically for the possibility of evaluating through the thickness shear deformation effects and axial membrane effects that were neglected in the use of thin wall Euler-Bernoulli beam bending kinematics. For this purpose, the Abaqus commercial finite element code was used and the PURC was modeled with Timoshenko beam theory based B22 elements. These beam elements are three noded quadratic beam elements and include transverse shear deformation and axial membrane deformation. The transverse shear deformation in these elements are treated as if the response were linear elastic, independent of the axial and bending responses. The circular PURC is modeled with 6 curved beam elements and the contact region is assumed to be a point.

The elastic moduli were numerically computed and the results so obtained are compared with our analytical predictions in Table 1 . This table also includes the results of the elliptical cell honeycombs that will be discussed in Sect. 3 .

In Table 1, we used measured material data ${ }^{3}$ to calculate the inplane elastic properties. The data are obtained through measurements related to real polycarbonate honeycomb cell specimens as reported in [10]. The agreement between the analytical prediction (column 2 of Table 1) and the numerical evaluation (column 3 of Table 1) is very strong.

\footnotetext{
3 'The Young's modulus of the polycarbonate material $E=2.4 G P a$, the Poisson's ratio of the polycarbonate material $\nu=0.3$, average thickness $t=0.0660 \mathrm{~mm}$, average radius $R=2.0394 \mathrm{~mm}$, minimum ellipticity $a / b=1.9978 \mathrm{~mm} / 2.0819 \mathrm{~mm}=0.9596$, average ellipticity $a / b=2.0825 \mathrm{~mm} / 1.9972 \mathrm{~mm}$ $=1.0427$ and maximum ellipticity $a / b=2.1515 \mathrm{~mm} / 1.9332 \mathrm{~mm}=1.1129$ where, $a$ and $b$ are the major and minor radii of the ellipse in the $X$ direction and $Y$ direction, respectively (see Fig. 4).
} 


\section{Inplane properties of perfect elliptical cell hexagonally packed honeycombs}

We now turn to the derivation of the inplane macroscopic elastic properties of a hexagonally packed elliptical cell honeycomb. Figure 4 describes a perfect PURC of the elliptic cell honeycomb and dimensions of the PURC. We leave the formulation quite general by considering perfect elliptical cells of major axis $2 a$ and minor axis $2 b$.

First, we consider the evaluation of $E_{x}{ }^{*}$. A free body diagram of the honeycomb for this case is shown in Fig. 5 a, where the nomenclature needed for the subsequent development is also indicated. The honeycomb is subjected to a macroscopic stress $\sigma_{x}$ in the $X$-direction. Since the cells are elliptic, an incremental arc length $d s$ along a cell segment is given in general by $d s_{1}=\sqrt{\left(a \cos \theta_{1}\right)^{2}+\left(b \sin \theta_{1}\right)^{2}} d \theta_{1}$ and $d s_{2}=\sqrt{\left(a \sin \theta_{2}\right)^{2}+\left(b \cos \theta_{2}\right)^{2}} d \theta_{2}$. Now, $M_{1}\left(\theta_{1}\right)$ $=-M_{a}-P_{2} b\left(1-\cos \theta_{1}\right)$ and $M_{2}\left(\theta_{2}\right)=M_{c}+P_{1} b \sin \theta_{2}$. Next, overall inplane moment equilibrium supplies

$M_{c}=\frac{1}{2}\left\{-2 M_{a}+P_{2} b(\sqrt{3}-2)-\sqrt{3} P_{1} b\right\}$.

The strain energy $U$ is given by

$U=2\left[\int_{0}^{\pi / 6} \frac{\left[M_{1}\left(\theta_{1}\right)\right]^{2}}{2 \hat{D}} d s_{1}+\int_{0}^{\pi / 3} \frac{\left[M_{2}\left(\theta_{2}\right)\right]^{2}}{2 \hat{D}} d s_{2}\right]$.

Invoking Castigliano's theorem provides the set of equations given in (2). Now, in this case,

$E_{x}^{*}=\frac{\sigma_{x}}{\varepsilon_{x}}=\frac{\left(P_{1}+P_{2}\right) / \sqrt{3} b}{\delta / a}$.

In general, this expression needs to be evaluated numerically in conjunction with the procedure that was described in conjunction with the circular cell honeycombs since the integrals associated with $U$ are not amenable to a closed form evaluation in view of the expressions for $d s_{1}$ and $d s_{2}$. However, in what follows, we show that for slight ellipticity as defined below, we can obtain closed form expressions for the macroscopic honeycomb properties. Thus, define a small parameter $\varepsilon$ for slight ellipticity in the following manner. Let $a=b(1+\varepsilon)$, where

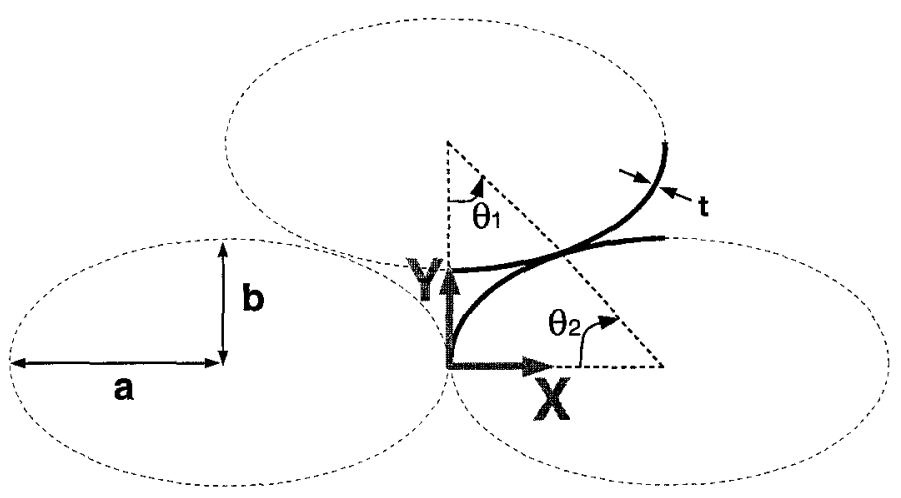

Fig. 4. A PURC of the elliptic cell honeycomb 


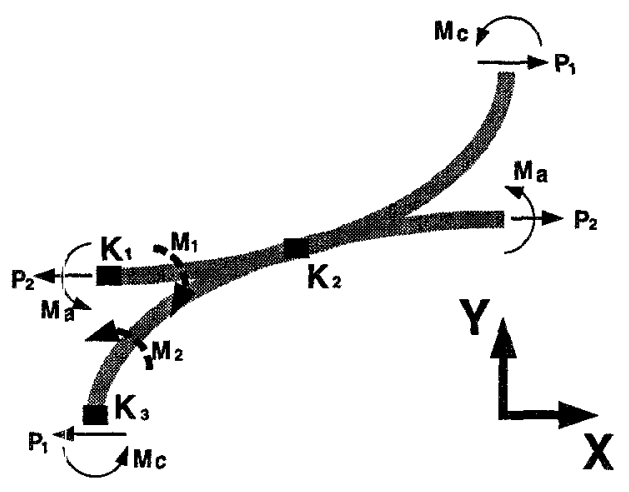

$\mathfrak{a}$

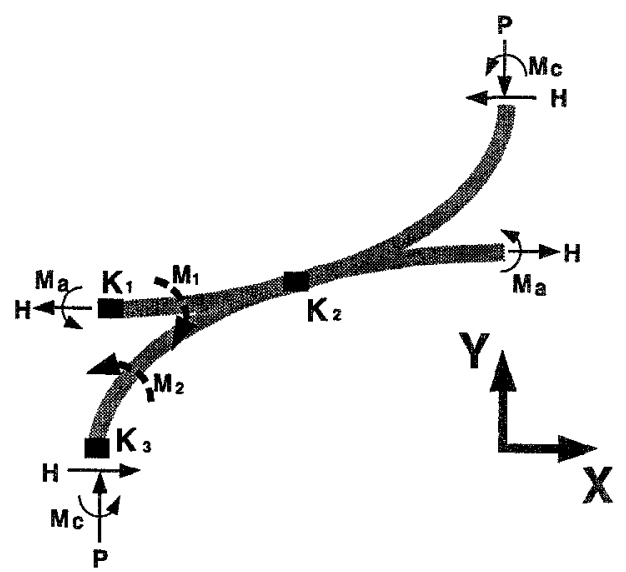

b

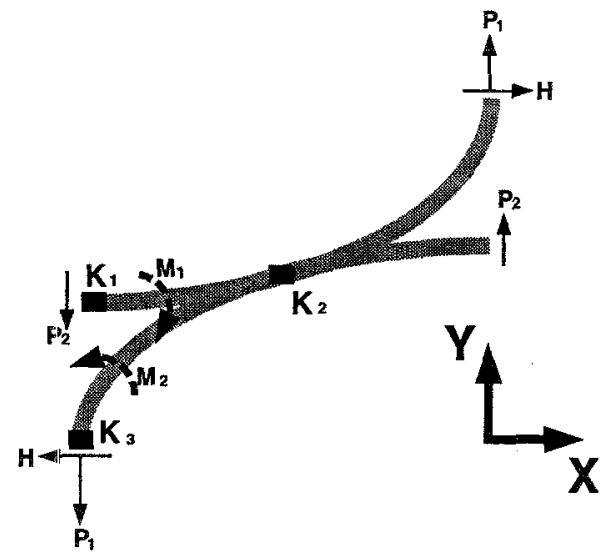

c

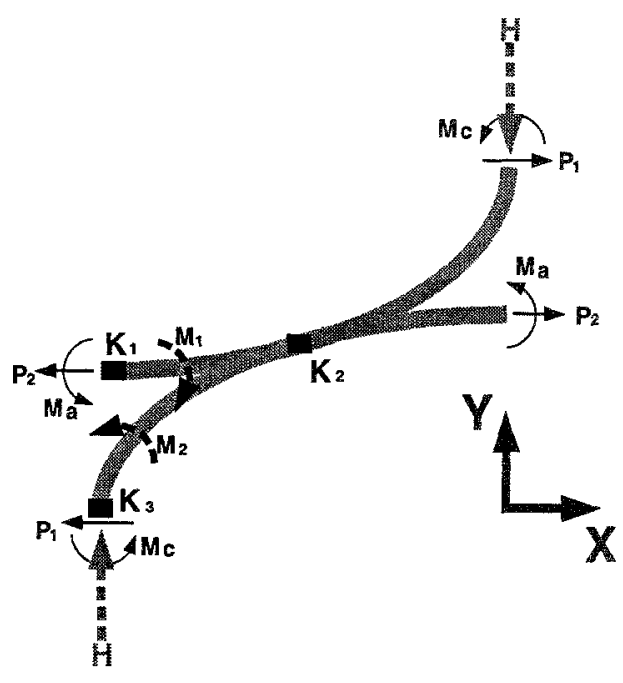

$d$

Fig. 5. a Free body diagram of elliptic PURC used to calculate the elastic modulus in the $X$-direction; b Free body diagram of elliptic PURC used to calculate the elastic modulus in the $Y$-direction; c Free body diagram of elliptic PURC used to calculate the shear modulus in the $X-Y$ plane; d Free body diagram of elliptic PURC used to calculate the Poisson's ratio in the $X-Y$ plane

$\|\varepsilon\| \ll 1$. Then,

$d s_{1}=\sqrt{\left(a \cos \theta_{1}\right)^{2}+\left(b \sin \theta_{1}\right)^{2}} d \theta_{1} \approx b\left(1+\frac{1}{2} \beta \cos ^{2} \theta_{1}\right) d \theta_{1}$,

and

$d s_{2}=\sqrt{\left(a \sin \theta_{2}\right)^{2}+\left(b \cos \theta_{2}\right)^{2}} d \theta_{2} \approx b\left(1+\frac{1}{2} \beta \sin ^{2} \theta_{2}\right) d \theta_{2}$,

where $\beta=2 \varepsilon+\varepsilon^{2}$. 
With these new approximations to $d s_{1}$ and $d s_{2}$, and performing the integrals associated with $U$ and using (2), the following closed form expression for $E_{x}{ }^{*}$ is obtained in terms of $\varepsilon=(a / b)-1$ :

$\frac{E_{x}^{*}}{E}=\frac{C_{1}(\varepsilon)}{\left(1-\nu^{2}\right)}\left(\frac{t}{b}\right)^{3}$

where $C_{1}(\varepsilon)=8.329+1.502 \varepsilon-4.366 \varepsilon^{2}+O\left(\varepsilon^{3}\right)$.

In terms of the relative density, the above equation becomes

$\frac{E_{x}^{*}}{E}=\frac{C_{11}(\varepsilon)}{\left(1-\nu^{2}\right)}\left(\frac{\varrho^{*}}{\varrho}\right)^{3}$

where $C_{11}(\varepsilon)=1.396+2.345 \varepsilon-1.401 \varepsilon^{2}+O\left(\varepsilon^{3}\right)$.

From the above two equations, it can be seen that when $\varepsilon \rightarrow 0$, we recover the corresponding results for the perfect circular cell case derived earlier.

To evaluate the macroscopic Young's modulus in the $Y$-direction, the honeycomb is subjected to a macroscopic stress $\sigma_{y}$ in the $Y$-direction and the corresponding free body diagram is as shown in Fig. 5 b. From overall inplane moment equilibrium,

$M_{c}=\frac{1}{2} P a-(\sqrt{3}-1) H b-M_{a}$.

The internal moments $M_{1}\left(\theta_{1}\right)$ and $M_{2}\left(\theta_{2}\right)$ are given by

$M_{1}\left(\theta_{1}\right)=M_{a}-H b\left(1-\cos \theta_{1}\right) \quad$ and $\quad M_{2}\left(\theta_{2}\right)=-M_{c}-H b \sin \theta_{2}+P a\left(1-\cos \theta_{2}\right)$.

The strain energy $U$ is given as before by expression (9). Substituting for $M_{1}\left(\theta_{1}\right)$ and $M_{2}\left(\theta_{2}\right)$ from (10) into (9) and invoking Castigliano's theorem provides the set of equations as given by (4). After a lengthy algebraic manipulation, and neglecting terms of $O\left(\varepsilon^{3}\right)$ and higher, the following expression for $E_{y}{ }^{*}$ is obtained.

$\frac{E_{y}{ }^{*}}{E}=\frac{C_{2}(\varepsilon)}{\left(1-\nu^{2}\right)}\left(\frac{t}{a}\right)^{3}$

where $C_{2}(\varepsilon)=8.329-4.421 \varepsilon+0.607 \varepsilon^{2}+O\left(\varepsilon^{3}\right)$.

Using the relative density, the above equation becomes

$\frac{E_{y}^{*}}{E}=\frac{C_{21}(\varepsilon)}{\left(1-\nu^{2}\right)}\left(\frac{\varrho^{*}}{\varrho}\right)^{3}$

where $C_{21}(\varepsilon)=1.396-2.835 \varepsilon+2.260 \varepsilon^{2}+O\left(\varepsilon^{3}\right)$.

Again, from the above two equations, it can be seen that when $\varepsilon \rightarrow 0$, we recover the corresponding results for the perfect circular cell case derived earlier.

The inplane shear modulus $G_{x y}^{*}$ was calculated by subjecting an elliptical PURC to a pure shear loading as was done for the circular cell case. The free body diagram corresponding to this case is shown in Fig. 5 c. Overall inplane moment equilibrium yields

$H=\frac{a}{\sqrt{3} b}\left(P_{1}+P_{2}\right)$.

The internal moments $M_{1}\left(\theta_{1}\right)$ and $M_{2}\left(\theta_{2}\right)$ are given by

$M_{1}\left(\theta_{1}\right)=P_{2} a \sin \theta_{1}$ 
and

$M_{2}\left(\theta_{2}\right)=H b \sin \theta_{2}-P_{1} a\left(1-\cos \theta_{2}\right)$.

Using Eq. (6) in conjunction with $U$ defined by (9) allows us to obtain $P_{1}$ and $P_{2}$ as linear functions of $\Delta$. Next, using the definition of macroscopic shear modulus

$G_{x y}^{*}=\frac{a}{\sqrt{3} b} \frac{\left(P_{1}+P_{2}\right)}{\Delta}$,

we obtain:

$\frac{G_{x y}^{*}}{E}=\frac{C_{3}(\varepsilon)}{\left(1-\nu^{2}\right)}\left(\frac{t}{a}\right)^{3}$

where $C_{3}(\varepsilon)=2.306+3.906 \varepsilon+0.808 \varepsilon^{2}+O\left(\varepsilon^{3}\right)$.

In terms of the relative density, the above equation reduces to

$\frac{G_{x y}^{*}}{E}=\frac{C_{31}(\varepsilon)}{\left(1-\nu^{2}\right)}\left(\frac{\varrho^{*}}{\varrho}\right)^{3}$

where $C_{31}(\varepsilon)=0.386+0.075 \varepsilon-0.557 \varepsilon^{2}+O\left(\varepsilon^{3}\right)$.

The above two equations, in the limit $\varepsilon \rightarrow 0$, lead to the corresponding results for the corresponding perfect circular cell case derived earlier.

Calculation of the macroscopic major Poisson's ratio $\nu_{x y}^{*}$ is similar to that of the circular cell case, whence an elliptic PURC is subjected to a macroscopic stress $\sigma_{x}$ in the $X$-direction and a virtual macroscopic stress $\sigma_{y}$ in the $Y$-direction. The corresponding free body diagram is as shown in Fig. $5 \mathrm{~d}$, where the virtual force $H$ due to $\sigma_{y}$ is indicated. Again, overall inplane moment equilibrium supplies

$M_{c}=-M_{a}-0.134 P_{2} b-0.866 P_{1} b-0.5 H a$.

The internal moments are given by

$M_{1}\left(\theta_{1}\right)=-M_{a}-P_{2} b\left(1-\cos \theta_{1}\right)$

and

$M_{2}\left(\theta_{2}\right)=M_{c}+H a\left(1-\cos \theta_{2}\right)+P_{1} b \sin \theta_{2}$.

The above quantities are substituted into (9) to evaluate $U$, after which Eq. (7) is used to solve for $P_{1}, P_{2}$ and $H$. Then, taking the limit $H \rightarrow 0$ and using the definition $\nu_{x y}^{*}=-\varepsilon_{y} / \varepsilon_{x}$ $=-(a /(\sqrt{3} b))(\Delta / \delta)$ yields

$\nu_{x y}^{*}=0.806+1.530 \varepsilon+0.654 \varepsilon^{2}+O\left(\varepsilon^{3}\right)$.

Once again, it can be seen that when $\varepsilon \rightarrow 0$, the corresponding result for the corresponding perfect circular cell case derived earlier is recovered.

\section{Discussion}

The results from a combined experimental and numerical study on the crushing response of honeycombs are reported by the authors in [10], [11]. In those studies, polycarbonate circular cell honeycombs were subjected to uniaxial compressive inplane loads and biaxial compressive 

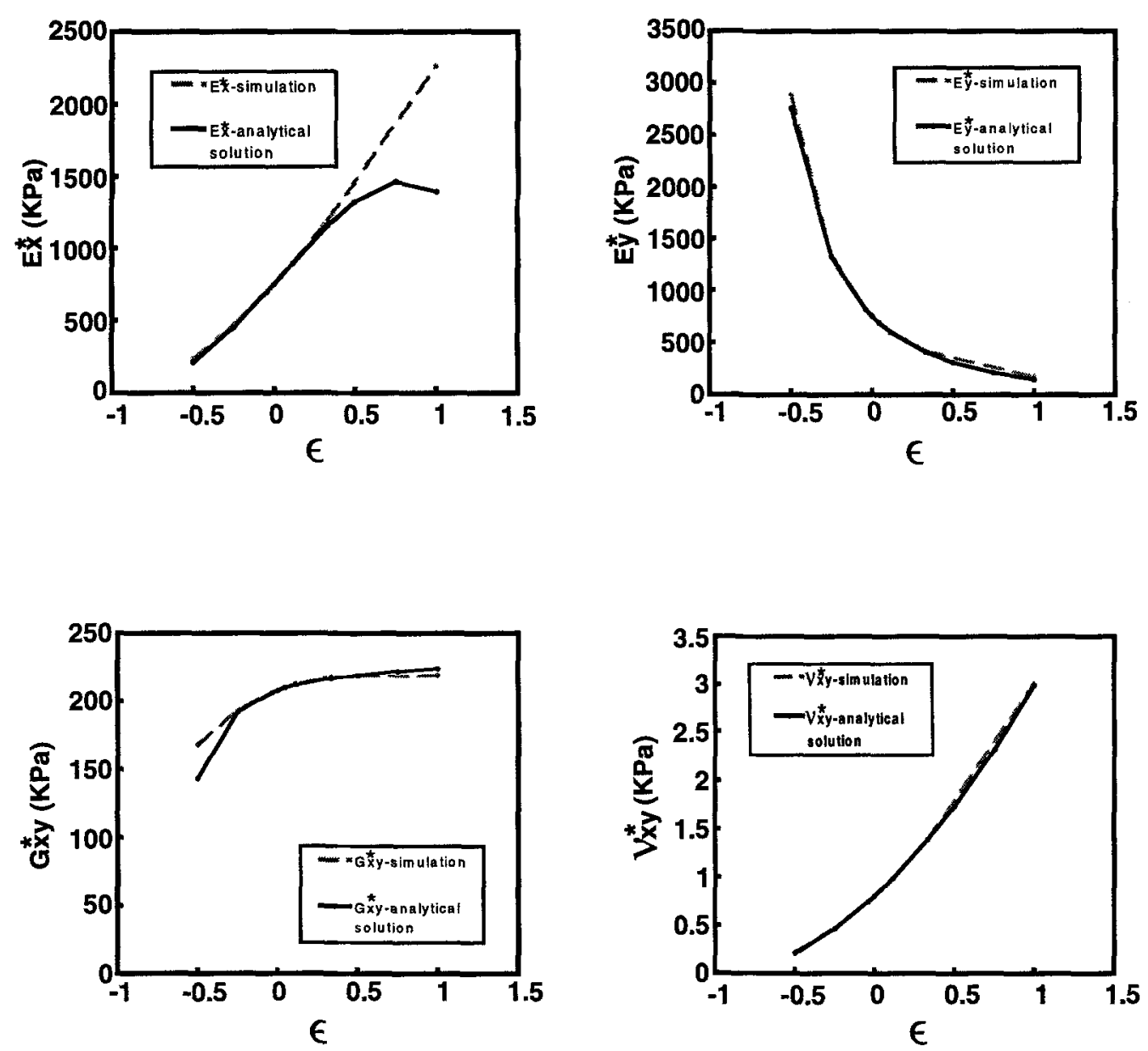

Fig. 6. Comparison of the inplane elastic properties for the honeycomb between the finite element simulation and the asymptotic analytical solution developed in the present paper, as a function of $\varepsilon$

inplane loads. Material properties and geometry of the cells in the honeycomb were measured via a high magnification optical microscope in conjunction with a digital data acquisition system. The authors found bounds for the cell ellipticity in terms of the ellipse aspect ratio $(a / b)$. These data were used in conjunction with the present analytical derivation to construct Table 1 which shows the range of linear elastic properties to be expected of these honeycombs for typical extremal and average values of $a / b$. As stated earlier, we also verified our analytical derivation numerically via the finite element method in which the honeycomb walls were modeled using Timoshenko beam theory based elements which include an approximate correction for the shear deformation through the thickness, and also include axial membrane deformation that was not included in our analytical derivation. These numerical results are also indicated in Table 1. As can be seen, the agreement between the analytical and numerical results are strong.

It must be noted that the analytical treatment pertaining to the elliptical cell case is quite general and is valid for any $a / b$ ratio. The boxed expressions we derived in closed form are clearly valid only for slight ellipticity when $\|\varepsilon\|=(a / b)-1 \ll 1$. For those situations when $\|\varepsilon\| \ll 1$, the evaluation of the elastic properties must be carried out numerically, where the integrals associated with the strain energy can be evaluated via numerical integration, for 
example, using the trapezoidal rule or the simpson's rule [12]. Alternatively, as was done in the present paper, a finite element based numerical simulation can be carried out for this purpose. Figure 6 shows a comparison between the finite element simulation based results and the analytically derived results for finite values of $\varepsilon$. From this figure, one can determine the range of validity (in terms of $\varepsilon$ ) of the asymptotic analytical predictions for the honeycomb inplane mechanical properties.

The expressions we have derived are for perfectly periodic circular cell or elliptical cell honeycombs. At present, we are investigating possible extensions of such derivations for the more practical case of non-periodicity [13], [14]. However, in these instances, if the number of cells in a macroscopic volume is large, we expect the above results to hold with sufficient accuracy for engineering applications. On the other hand, accurate calculations of the near constant plateau load when compressive inplane crushing leads to cell buckling is quite a challenging task [3]. Gibson and Ashby [1] have indicated an approximate procedure for calculating the inplane compressive plateau load for hexagonal cell honeycombs based on Euler strut buckling with a suitable end constraint factor to account for rotational stiffness at cell wall joints. They report good agreement of their calculations checked against experiments conducted with relatively thick walled rubber honeycombs [15], [16]. This aspect of the problem as it relates to the present investigation is a subject of a separate study [10], [11].

\section{Concluding remarks}

The study of inplane properties of honeycombs is important because they exhibit and highlight the mechanisms by which such solids deform and fail when subjected to inplane loads. In this paper, the authors have presented a derivation of the inplane elastic properties of a class of circular cell and elliptical cell hexagonally packed honeycombs. The derived analytical expressions have been checked against a finite element analytical model that included transverse shear deformation and axial membrane deformation. For elliptical cell honeycombs, closed form expressions for the macroscopic inplane elastic constants in terms of the material properties, cell wall thickness and a small parameter $\varepsilon$ signifying slight ellipticity of the cells has been derived. The corresponding general expressions for any value of $\varepsilon$ are also shown and these need to be evaluated numerically. The elliptic cell results are shown to reduce to the corresponding circular cell case when $\varepsilon \rightarrow 0$.

\section{Acknowledgement}

We are grateful to the Aerospace Engineering Department, University of Michigan, for financial support (JC). AMW is grateful to Prof. N. Triantafyllidis for bringing to his attention, in 1991, the early work by Gibson and Ashby (reference [17] in the bibliography).

\section{References}

[1] Gibson, L. J., Ashby, M. F.: Cellular Solids: Structure and Properties. Oxford: Pergamon Press, 1988.

[2] Warren, W. E., Kraynik, A. M.: Nonlinear elastic behavior of open-cell foams. J. Appl Mech Trans ASME 58, 376-381 (1991). 
[3] Papka, S. D., Kyriakides, S.: In-plane compressive response and crushing of honeycomb. J. Mech. Phys. Solids 42, 1499 - 1532 (1994).

[4] Papka, S. D., Kyriakides, S.: In-plane crushing of a polycarbonate honeycomb. Int. J. Solids Structures 35, 239-267 (1998).

[5] Klintworth, J. W., Stronge, W. J.: Elasto-plastic yield limits and deformation laws for transversely crushed honeycombs. Int. J. Mech. Sci. 30, 273-292 (1988).

[6] Triantafyllidis, N., Schraad, M. W.: Onset of failure in aluminum honeycombs under general inplane loading. J. Mech. Phys. Solids 46, $1089-1124$ (1998).

[7] Torquato, S., Gibiansky, L. V., Silva, M. J., Gibson, L. J.: Effective mechanical and transport properties of cellular solids. Int. J. Mech. Sci. 40, $71-82$ (1998).

[8] Simone, A. E., Gibson, L. J.: Effects of solid distribution on the stiffness and strength of metallic foams. Acta Materialia 46, $2139-2150$ (1998)

[9] Gere, J. M., Timoshenko, S. P.: Mechanics of Materials. 2nd ed., 1984.

[10] Chung, J., Waas, A.: Collapse, crushing and energy absorption of circular-celled honeycomb and sandwich panels. AIAA-CP99-1358, 1999.

[11] Chung, J., Waas, A.: Compressive response of circular cell polycarbonate honeycombs under inplane biaxial stresses, accepted for publication, ASCE J. of Engineering Mechanics, 2000.

[12] Faires, J. D., Burden, R.: Numerical Methods. 2nd ed., 1998.

[13] Silva, M. J.,Gibson, L. J., Hayes, W. C.: The effects of non-periodic microstructure on the elastic properties of two dimensional cellular solids. Int. J. Mech. Sci. 37, 1161-1171 (1995).

[14] Silva, M. J., Gibson, L. J.: The effects of non-periodic microstructure and defects on the compressive strength of two-dimensional cellular solids. Int. J. Mech. Sci., (forthcoming) 1996.

[15] Gibson, L. J., Engineering Ph. D. thesis, Engineering Department, Cambridge Univ., Cambridge 1981.

[16] Gibson, L. J., Ashby, M. F., Schajer, G. S., Robertson, C. I.: Mechanics of two-dimensional cellular materials, Proc. R. Soc. Lond., Ser. A 382, 25-42 (1982).

[17] Gibson, L. J., Asby, M. F.: The mechanics of three-dimensional cellular materials. Proc. R. Soc. A 382, $43-59$ (1982).

Authors' address: J. Chung and A. M. Waas, Dept. of Aerospace Eng., The University of Michigan, Ann Arbor, MI 48109-2118 (E-mail: chunga@engin.umich.edu; dcw@engin.umich.edu) 